To be published in Journal of Optical Communications and Networking:

Title: $\quad$ Flexible coherent UDWDM-PON with dynamic user allocation based on limitedtunability lasers

Authors: Josep Segarra,Vicent Sales,Victor Polo,Jeison Tabares,Josep Prat Gomà

Accepted: 14 May 20

Posted 15 May 20

DOI: $\quad$ https://doi.org/10.1364/JOCN.391023

(C) 2020 Optical Society of America 


\title{
Flexible coherent UDWDM-PON with dynamic user allocation based on limited-tunability lasers
}

\author{
Josep Segarra* , Vicent Sales, Víctor Polo, Jeison tabares and Josep Prat
}

Universitat Politècnica de Catalunya (UPC), Signal Theory and Communications Department (TSC), Barcelona, E-08034

*Corresponding author: jsegarra@tsc.upc.edu

Received 18 February 2020; revised XX Month, XXXX; accepted XX Month XXXX; posted XX Month XXXX (Doc. ID XXXXX); published XX Month XXXX

Coherent WDM technologies have leveraged the optical communication systems in core networks, increasing the fiber capacity by transmission with advanced modulation formats and mitigation of impairments with digital signal processing (DSP). However, these solutions are too expensive for access networks, where cost, power budget and footprint are limited. Hence, the key technology will be developing low-cost coherent transceivers providing an excellent selectivity, and giving high sensitivity, which allows high splitting ratios. This article reports an experimental design of a low-cost coherent ultra-dense WDM-PON (UDWDM-PON) with 6.25 GHz channel spacing. The users' optical network unit (ONU) is built employing coherent transceivers with two paired low-cost DFB lasers, one as local oscillator and another as transmitter, offering simplicity and low-cost hardware; likewise, the optical line terminal (OLT) at central office can profit from the same design. The ONU DFB lasers have wavelengths with limited thermal tunability, controlled by a thermo-electric cooler (TEC), which is used to allocate the wavelengths. A medium access control (MAC) at the OLT manages the spectrum channel allocation for ONUs demanding connection when activation is requested: the OLT furnishes an optical carrier wavelength for the ONU to obtain connection by a control algorithm, assigning a down-channel and another paired up-channel assigned to the ONU DFB transmitter. The MAC can reassign the channels because of interference or collision in a dynamic wavelength allocation (DWA). Measures in an activation process and in channel reassignment have been performed in environment conditions, including control signals and physical parameters of DFB lasers, demonstrating the practical viability of the PON scaling from 32 up to 256 wavelength channels.

http://dx.doi.org/10.1364/JOCN.99.099999

\section{INTRODUCTION}

Current optical access networks standards and developed fiber-to the-home (FTTH) technologies need to evolve to face the upcoming bandwidth (BW) growth driven by multimedia services with high definition video, cloud-computing, business services, and emerging next generation mobile networks like $5 \mathrm{G}$. The passive optical networks (PONs), installed with a fiber and split-built optical distribution network (ODN), are deploying the 10GE-PON [1] and XG-PON [2] standards, with $10 \mathrm{~Gb} / \mathrm{s}$ data rate and time division multiplexing (TDM). Though, they employ only two wavelengths ( $\lambda s)$, thus with poor use of the optical fiber spectrum, whereas core networks are now reaching its limits [3]. To enhance the PON capacity, the next generation NG-PON2 recommendation [4] gathers from 4 to 8 XGPONs on a time and wavelength division multiplexing (TWDM) basis and aggregate capacity up to $80 \mathrm{~Gb} / \mathrm{s}$, coexisting with legacy systems through $\lambda$ overlay and keeping the split-based ODN, while still using the intensity-modulation and direct-detection (IM-DD) transmission format. However, scaling TWDM to higher bit rates may not be compatible with low-cost photonic devices, explicitly the bit rate per $\lambda$, with high hardware cost and excessive energy consumption.

On the other hand, several WDM-PONs have been introduced making also use of IM-DD formats, and mainly based on deploying arrayed waveguide gratins (AWGs) in the ODN. An automatic wavelength allocation method was proposed, based on maximization of the beating noise between a seed light from the OLT at central office (CO) and a tunable laser (TL) at the ONU [5]; however, requiring a broadband light source at the OLT, which is filtered by the AWG, increases complexity and costs. Another proposal for automatic wavelength control is based on the detection of backscattered light [6], where the light generated by the ONU TL passes through the AWG twice and is back received at the ONU, then the $\lambda$ of the ONU TL is matched at the maximum power of the received $\lambda$, corresponding to the peak center of the AWG channel. In an alternative method, a centralized control by the OLT is facilitated by imprinting feedback of the received power of each ONU upstream by the OLT to enable calculation of relative $\mathrm{ONU} \lambda$ deviation; this feedback is transferred by the OLT to the ONUs by pilot tones or a MAC $[7,8]$. These WDM-PONs make use of expensive full TLs in the optical band and of AWGs in the ODN, which is not compatible with legacy systems based in power splitters and decreases granularity. Nevertheless, a colorless and pugand-play WDM access over existing power splitter infrastructure has also been presented [9]; it makes use again of full TLs and an automatic $\lambda$ control at the $\mathrm{ONU}$, which is based on a tunable filter (TF) exploring the full band until sensing a downstream unused $\lambda$, where the TF is set, and next a paired $\lambda$ upstream is automatically assigned, according to a 
wavelength assignment table indicating the combination of the corresponding downstream and upstream $\lambda$ s.

Our proposal is a novel coherent UDWDM-PON making use of a wavelength-to-the-user (WTTU) scheme, furnishing each customer a single $\lambda$, while keeping coexistence with legacy systems and spacing channels densely at a few $\mathrm{GHz}$, thus achieving a high spectral efficiency [10]. Moreover, the UDWDM-PON can be implemented with affordable low-cost components [11] and also be flexible in user BW and channel allocation [12,13]. Considering ITU recommendations, the grid will evolve to finer $12.5 \mathrm{GHz}$ slots [14], so the next step can be down to 6.25 $\mathrm{GHz}$, enabling a PON for high and dense number of users. Additionally, superior bit rates per $\lambda$ can also be achieved exploiting low-cost distributed feedback (DFB) lasers, photonic integration, simplified direct optical modulation, consumer electronics and low-complexity digital signal processing (DSP) $[15,16]$.

The developed UDWDM-PON under the EU FP7 COst-effective COhereNt Ultra-dense-WDM-PON for lambda-To-the-user access (COCONUT) project $[10,11]$, was a progress in order to support new emerging customer and mobile front-haul and back-haul applications. Novel low-cost coherent techniques improve the sensitivity to about $50 \mathrm{dBm}$, thus reaching a higher power budget (>45dB) and hence, serving higher number of users (up to 256) at long distances (up to 60 $\mathrm{km}$ ), being each user served with flexible data rates from $1.25 \mathrm{~Gb} / \mathrm{s}$ to $10 \mathrm{~Gb} / \mathrm{s}[15,16]$.

When implementing an UDWDM-PON, for each ONU, a TL is needed as local oscillator (LO) for coherent reception and another TL for transmission, and two different $\lambda s$ are used for down and up transmission to avoid Rayleigh backscattering (RB) at the single-fiber PON transmission. The tuning range of the lasers is a key parameter for achieving a low-cost implementation of the ONU; such narrow channel spacing enables to choose simple DFB lasers, with limited tunability of only about $\pm 1.6 \mathrm{~nm}$ by simple temperature control $[17,18]$; VCSEL lasers could be similarly used, with lower performances. Employing DFB-lasers in direct modulation and coherent detection schemes has been shown [19]. When DFB-lasers are manufactured, they exhibit random nominal $\lambda s$ with statistical Gaussian distribution over few nanometers range [17]; but this range can be extended to a larger optical band, of e.g. 10 to $40 \mathrm{~nm}$, merely by merging lasers with different nominal $\lambda s$ in a number of sub-bands and, hereafter, the random $\lambda$ s gathered in a full optical band have approximately a new statistical uniform distribution [20]. When the TLs sources at the ONU, with very limited thermal tunability used to allocate $\lambda$ s avoiding collisions, have random nominal $\lambda$ s distributed in a band, we apply a statistical UDWDM allocation approach [20]. Hence, to efficiently distribute the spectrum in activation and also during operation, dynamic wavelength allocation (DWA) algorithms using the limited thermal tunability schemes were proposed $[20,21]$.

In this work we present an experimental demonstration of an UDWDM-PON, by furnishing a WTTU approach and also introducing the proposed architectures for the ONUs at users' premises and for the OLT at the CO. DWA schemes in activation and also in operation processes are proposed to estimate the needed optical BWs. Afterwards, a trial setup is introduced to show a network system implementation, including ONU wavelength assignment, activation and also operation, which are all managed by a medium access control (MAC) in the OLT.

This article is organized in the following sections: Section 2 presents the network architectures and DWA algorithms. In Section 3 the trial setup is introduced, exposing the physical system characteristics, the managing and control functions for activation and the wavelength assignment. In Section 4 the experimental results are reported, and, finally, in Section 5 the conclusions are discussed.

\section{UDWDM-PON ARCHITECTURES}

In the proposed architectures, the key technology will be low-cost coherent transceivers. At the ONU side, employing TLs based on cheap DFB-lasers, and at the CO part, the OLT implementation will reproduce the same ONU design for each customer. The ODN is built with optical fibers and splitters, coexisting with legacy systems.

\section{A. ONU architectures}

The ONU implementation is based on coherent detection, using two TLs, one as LO for reception and another as transmitter, with direct or external modulation and two different $\lambda s$ in separated down and up bands to avoid RB crosstalk (Fig. 1). The TLs are of low-cost DFB type with limited tunability adjusted by heating or cooling and with nonpreselected nominal $\lambda s$, which have statistical uniform distribution in the PON optical band. DFB-lasers have a constant linear variation of $\lambda$ with temperature of about $0.1 \mathrm{~nm} /{ }^{\circ} \mathrm{C}$ highly reproducible between 14 to $55^{\circ} \mathrm{C}$ [21], covering $3.2 \mathrm{~nm}$, thus tuning up to 64 channels of 6.25 $\mathrm{GHz}$, within the $30 \mathrm{~nm}$ of C-band. The temperature range is easily reached with a thermo-electric cooler (TEC) or Peltier.

A particular case, that simplifies the $\lambda$ control, is having the ONU TLs paired from the factory, with a fixed $\lambda$ difference between their nominal $\lambda s$. When a TL moves the tuning, the other paired TL moves its $\lambda$ in the same way; this will provide an easy activation process, which will be profited in the trial setup. Several types of integrated dualwavelength semiconductor tunable lasers have been reported: DFB [22,23], VCSEL [24], Fabry-Pérot [25] and, besides, a direct-modulated dual-wavelength laser [26], realized by coupling two DFBs, demonstrating their feasibility in integrated photonics.

Direct DFB phase modulation in Fig. 1 is obtained by driving the laser with the high-pass equalized data, taking advantage of the laser chirp. Directly phase modulated DFB lasers transmitting from 1.25 to 10 $\mathrm{Gb} / \mathrm{s}$, with modulations formats spanning from DPSK to 8-(A) PSK, have been reported with simplified coherent detection $[16,19]$. The receiver optical front-end implements $3 \times 3$ fused couplers with 6 photodiodes (PDs) for coherent homodyne detection [10], and two extra options for heterodyne detection: $3 \times 3$ coupler with 3 PDs or 2x2 coupler with 4 PDs [27]. All the receivers feature polarizationindependent coherent detection. These coherent transceiver architectures are simple and low-cost, avoiding external Mach Zehnder (MZ) modulators, tunable lasers and optical $90^{\circ}$ hybrids.

When using coherent homodyne detection with a bit rate of 1.25 $\mathrm{Gb} / \mathrm{s}$, the channel spacing was taken to be $6.25 \mathrm{GHz}$ [28,29], allocated in C-band. A guard band of about $3 \mathrm{GHz}$ is left, since the minimum wavelength separation between two adjacent channels is $3 \mathrm{GHz}$ [28].

Furthermore, recently, to flexibly adapt to different bit rates with complexity-reduced coherent transceivers, transmissions up to 10 $\mathrm{Gb} / \mathrm{s}$ by direct amplitude-and-phase modulation of dual electroabsorption modulated laser (EML), have been shown $[15,16]$, with different modulation formats and BWs.

Alternatively, a DPSK coherent heterodyne detection at the ONU can be implemented with two photodiodes (PDs), and the up DPSK modulation at the ONU can be realized by injecting data to a simple reflective semiconductor optical amplifier (RSOA), transmitting at 1.25 $\mathrm{Gb} / \mathrm{s}$ and with $12.5 \mathrm{GHz}$ total BW [11,30]. 


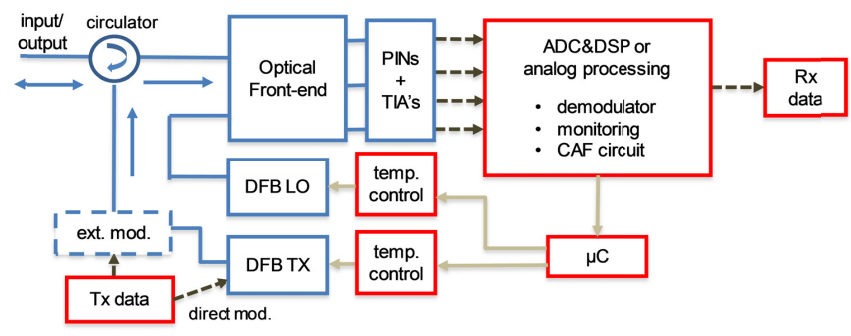

Fig. 1. ONU transceiver block diagram with coherent detection, using two TLs with external or with direct modulation.

\section{B. OLT architectures}

The OLT design is also based on coherent transceivers, serving all deployed ONUs in the PON [20]. The simplest architecture is realized with power splitters without any filtering, whenever using a different or the same band for upstream and downstream. Another design is based on 1xM-ports AWGs; in order to have full spectral $100 \%$ efficiency, the use of an athermal AWG combined with a thermal AWG was proposed, interleaving its spectral tuned response.

An optical spectrum monitor block is introduced in the OLT to continuously track all $\lambda s$ present in the PON and manage them in power and allocation, during activation processes and also in operation; the OLT controller makes decisions about reassignments, when needed, to deal with environmental conditions, by using DWAs schemes [20].

\section{Wavelength channel assignment}

At the ONU, the limited thermal tunability of the DFB lasers in a spectral tunable window (TW) is used to provide a WTTU service. The ONU DFBs have random $\lambda$ s distributed uniformly in the PON optical band and wavelength channels are assigned in the TWs without collision in activation and in operation processes, in a Statistical UDWDM strategy.

The OLT controls the wavelength allocation providing a channel in activation and in operation, but also a channel reassignment when needed because of: environmental changes affecting the TW, interference or ONU BW change in a flexible spectrum allocation.

Initially, two wavelength assignment (WA) paradigms managing the activation and operation were foreseen [31]:

- Static wavelength assignment (SWA); there, once wavelengths are assigned they remain static.

- Dynamic wavelength assignment (DWA); there, wavelengths reallocations are made, to even satisfy network changes in environmental temperature conditions.

These paradigms are combined with heuristic assignment schemes to practically distribute the ultra-dense spectrum. Three heuristic channel assignment algorithms have been proposed to avoid contention collision and efficiently organize the spectrum, suited for the Statistical UDWDM-PON scenario with limited tunability [20,21]:

- First fit (FF), allocating the free channel closer to the random ONU wavelength.

- Maximum scattering (MS), selecting the free channel providing maximum distance between two busy channels.

- Maximum admittance (MA), assigning the free channel giving the maximum options of admission when a future new request arrives at the same assigned channel.

To find the total needed optical BW, expressed in number of channels, the proposed heuristic assignment schemes (FF, MS and MA) were evaluated by obtaining the probability of an entrant ONU to be refused (ONU rejection probability), through simulations with several ODN splits [20].

Statistical dimensioning was realized for different numbers of users and for the different scenarios considered: channel BWs of $6.25 \mathrm{GHz}$ or $12.5 \mathrm{GHz}$, for homodyne or heterodyne, and homodyne paired TLs cases. Table I summarizes the required number of channels and bands for the different cases, using DWA with the best MA scheme, and considering an $\mathrm{ONU}$ rejection probability of $0.1 \%$ [21]. If the operator would prefer to avoid any rejection for some priority users, it could be solved in a practical deployment by provisioning such users with an ONU at a "safe" $\lambda$ or with fully tunable lasers. The related cost can be sufficiently compensated by the avoidance of the complex multielectrode widely tunable lasers at ONUs.

TABLE I

REQUIRED CHANNELS IN ACTIVATION PROCESS

Homodyne Heterodyne \& homodyne

(6.25 GHz/channel) paired TLs (12.5 GHz/ch.)

\begin{tabular}{cccc} 
Users & 2 TLs, 2 bands & 2 TLs, 1 band & 1 TL \& 2 TLs, 1 band \\
\hline 32 & $2 \times 32$ & 68 & 34 \\
64 & $2 \times 68$ & 140 & 75 \\
128 & $2 \times 141$ & 287 & 156 \\
256 & $2 \times 288$ & 583 & 317 \\
\hline \hline
\end{tabular}

\section{PROPOSED PON SETUP}

\section{A. Network parameters and managing for activation}

The physical parameters and managing controls in the setup for activation and operation with DWA are:

- Each ONU is equipped with two DFBs, one for down TX as LO and another for up TX, that are originally paired with a fixed $\lambda$ separation of $50 \mathrm{GHz}$; so, when the LO is synchronized at an assigned down-channel, the transmitter TL is also synchronized at a paired up-channel, hence making the activation process simpler and fast. This separation is kept constant over the tuning range with a tolerable error. This proposed arrangement does not break the condition of $\lambda$ agnosticism of the ONUs at the user provisioning, as it can take any ONU randomly.

- The ONU TLs are DFBs tuned thermally with a TEC control circuit, providing a variation of $0.1 \mathrm{~nm} /{ }^{\circ} \mathrm{C}$ typically; thus, with a $\pm 16{ }^{\circ} \mathrm{C}$ deviation from its nominal temperature, we tune a defined basic band of $400 \mathrm{GHz}$ in C-band. This basic tuning band is divided in 8 sub-bands of $50 \mathrm{GHz}$, with 4 sub-bands for down $(200 \mathrm{GHz})$ and 4 interleaved contiguous sub-bands for up (200 GHz); thus, each $50 \mathrm{GHz}$ sub-band can have 8 down/up channels of $6.25 \mathrm{GHz}$ (Fig. 2). Using different subbands for down/up mitigates the single-fiber RB.

- The channel spacing for down/up transmission at $1.25 \mathrm{~Gb} / \mathrm{s}$ with direct phase modulation is of $6.25 \mathrm{GHz}$ for homodyne coherent detection. Moreover, the channelization is flexible, allowing higher rates with other modulation formats and down/up BW channels of 12.5, 25 and up to $50 \mathrm{GHz}$, and also heterodyne detection [30].

- This down/up sub-band organization is also useful for the OLT design with $100 \mathrm{GHz}$ port AWGs [20]; hence, an AWG can be devoted to down TX and the other paired AWG, tuned at an offset of $50 \mathrm{GHz}$, for up RX. The separation of the paired DFBs at the ONU is also $50 \mathrm{GHz}$, and both lasers tune the complete basic band. The DFBs can work into the range of $18{ }^{\circ} \mathrm{C}$ to $50^{\circ} \mathrm{C}$, thus preserving the modulation quality at the TX side.

- This basic network segment of $400 \mathrm{GHz}$ allocates a maximum of 32 users with center wavelengths falling in this band, spaced by $6.25 \mathrm{GHz}$ (Fig. 2). The nominal $\lambda$ specified at $25^{\circ} \mathrm{C}$ of the LO DFB will be at the center of the band of $400 \mathrm{GHz}$ minus $100 \mathrm{GHz}$, which is at $100 \mathrm{GHz}$ from the beginning of the band, and the paired nominal $\lambda$ of the TX DFB just $50 \mathrm{GHz}$ upper. Once the LO DFB and the TX DFB are biased, their 
temperature naturally grows $10^{\circ} \mathrm{C}$ approximately without TEC activation, and their $\lambda$ s move $100 \mathrm{GHz}$ upper, reaching $200 \mathrm{GHz}$ and $250 \mathrm{GHz}$ from the beginning of the band, respectively.

- This pattern can be extended modularly to multiples of 32, suiting ODNs of 64 and up to 256 splits. In this extended network, the ONU nominal $\lambda s$ of the paired TLs are assigned to the corresponding basic band module of 32-split where each ONU is collocated.

- In this way, in the enlarged network for more than 32 users, the paired $\lambda s$ can also be non-preselected, having random $\lambda s$ with uniform distribution in the extended band; then, the statistical rules about ONU rejection for the homodyne paired TLs apply (Section II). Hereafter, the extended optical bandwidth has to be increased few sub-bands on the right and on the left of the band for the rejection probability of $0.1 \%$, with a number of channels as reported in Table I: 317 paired channels are required to allocate the 256 random ONUs, that is $32 \mathrm{~nm}$ approximately (16+16 for down+up stream) (Fig. 2).
- The MAC at the CO manages the spectrum channel allocation of the ONUs: the OLT always provides a carrier wavelength without data modulation, which will be available for the entrant ONU to get down connection at an assigned down-channel in its tunable band. The real $\lambda$ of the LO DFB may come shifted some $\mathrm{GHz}$ from its center wavelength due to a production drift; hence, two stable carrier wavelengths are furnished by the OLT to guarantee that any ONU can tune at least one; one situated from the start of the tuning band until $150 \mathrm{GHz}$, where there is the nominal $\lambda$ of the LO DFB, and the other from $200 \mathrm{GHz}$ to $350 \mathrm{GHz}$, both from the band start. Hereafter, the LO DFB is tuned to one of these carriers, by sweeping its temperature depending on its TW capability, and a down-channel is assigned to the ONU. These carriers will be provided by the OLT meanwhile there are free channels in the band.

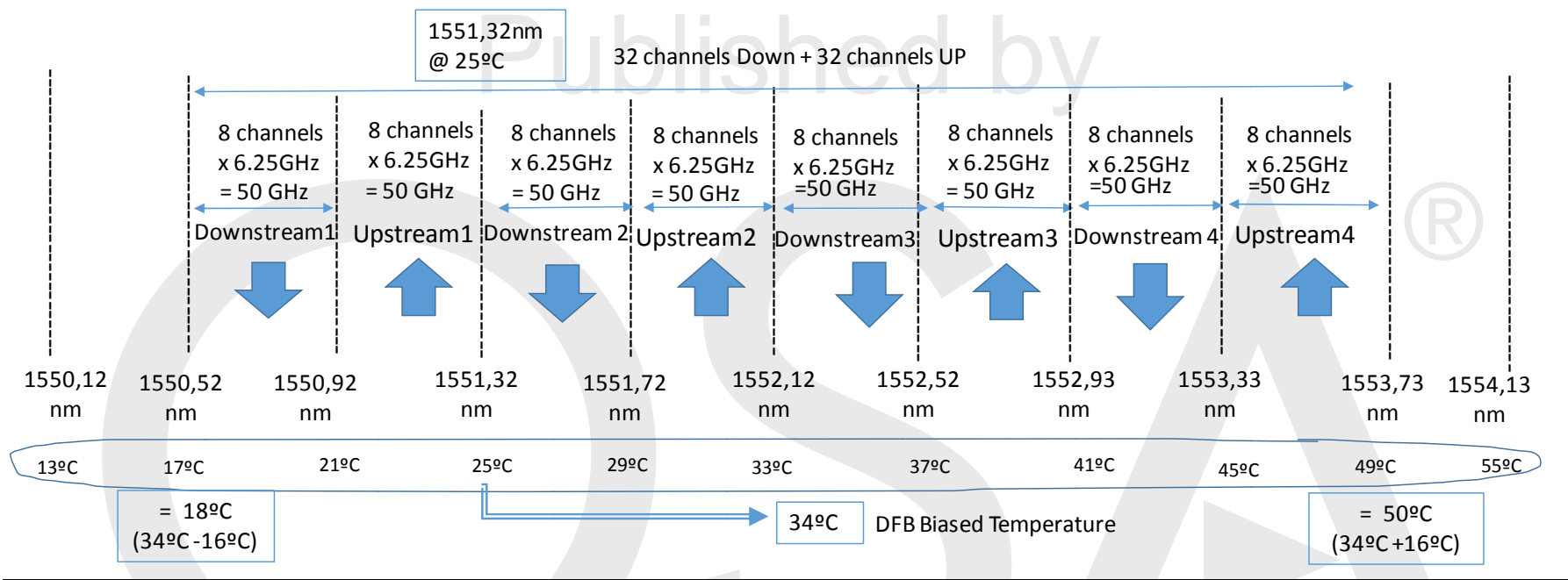

Fig. 2. Basic optical band of $400 \mathrm{GHz}$ organized in down/up sub-bands of $50 \mathrm{GHz}$ for 32 users spaced by $6.25 \mathrm{GHz}$, matching DFB tunable lasers.

- The ONU has a monitoring circuit to detect the unmodulated carrier provided by the OLT, where the LO DFB will be assigned to demodulate the down channel. Accordingly, the paired TX DFB is tuned and blindly allocated to the free paired assigned up-channel. The DFB TX is tuned at low power, experimentally found to be $18 \mathrm{~dB}$ less than the operating power in order not to disturb the working channels. Once the allocation is completed, the TX DFB power is increased to the operating power. A specific function, related to dynamic power control, is described in next sub-section. The ONU control is always monitoring in order to have fast response to the OLT MAC actions.

- The MAC can force the working ONUs to change and reassign the connected channels because of channel interference, collision or traffic demands in a DWA procedure.

- For the experimental demonstration, the nominal DFB laser is between the channels 31 and 32 of the $100 \mathrm{GHz}$ ITU grid. The commercial DFBs have been chosen at $1551.72 \mathrm{~nm}$ nominal $\lambda$ at $25^{\circ} \mathrm{C}$, reaching $34^{\circ} \mathrm{C}$ when biased and tuned to $1552.32 \mathrm{~nm}$, in the centre of the band; hence, considering a limited tunability of $\pm 1.6 \mathrm{~nm}\left(0.1 \mathrm{~nm} /{ }^{\circ} \mathrm{C}\right)$, the DFB can work in the eight $50 \mathrm{GHz}$ sub-bands: down/up, covering 32 channels of $6.25 \mathrm{GHz}$ for up and other 32 channels for down. The temperature range goes from $18{ }^{\circ} \mathrm{C}$ to $50{ }^{\circ} \mathrm{C}$, below the $55{ }^{\circ} \mathrm{C}$ maximum working temperature. As we do not dispose of integrated paired DFBs, the LO DFB and the TX DFB are identical, assembled in the ONU box.
- The DFBs have been mounted in a board (Fig. 3), supporting the required functionalities, like: bias current internal/external control for power laser managing, temperature control for frequency allocation and stabilization, RF input data equalization for direct modulation, analogue input/output ports for external control and monitoring.

- The receiver for homodyne testing has been realized with digital real-time processing after sampling the data recovered by PDs with ADCs operating at the symbol rate and processed by FPGA [32].

- The heterodyne receiver is implemented with off-the-shelf analogue components in a micro-strip board (Fig. 3), supporting: polarization independency, DPSK demodulation, frequency deviation measurement for LO automatic frequency control (AFC), power detection tracking, and analogue data output ports.

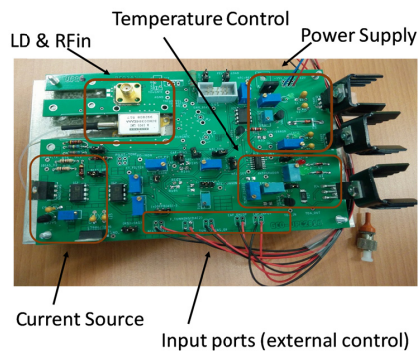

(a) 


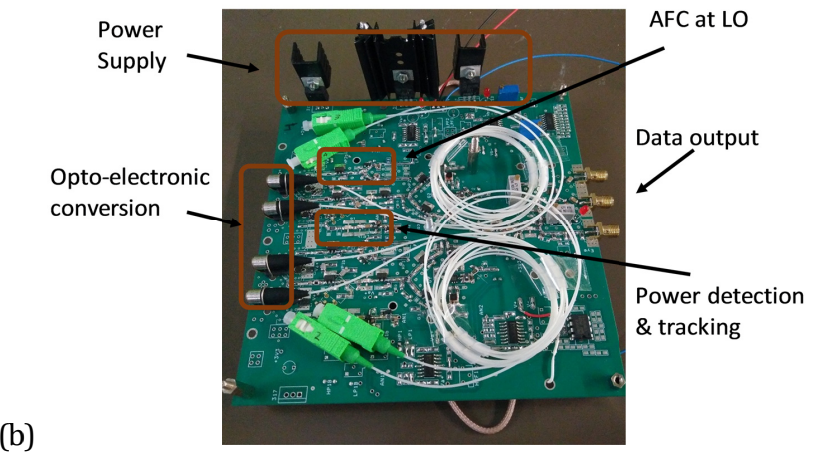

Fig. 3. (a) The implemented DFB-laser on a $150 \mathrm{~mm}$ x $100 \mathrm{~mm}$ board and (b) the heterodyne receiver on a $150 \mathrm{~mm} \times 150 \mathrm{~mm}$ board.

\section{B. Functions for channel activation}

During the activation of a new entrant ONU, the reception part of the ONU performs: the LO wavelength reset and initialization from the beginning of the band, the optical band scanning until an OLT carrier to be assigned is found, and the final allocation. The TX tracks similarly at $50 \mathrm{GHz}$ shift.

Some specific functions are used for achieving this activation:

- 'BM' (band monitoring): the ONU LO sweeps the band homodyning with a $150 \mathrm{MHz}$ tracking filter and zero-bias power detector, searching for the OLT carrier where to be allocated. This works as a high resolution optical spectrum analyzer (HR-OSA), covering $3.2 \mathrm{~nm}$ in 10 $\mathrm{s}$ with a $\pm 16{ }^{\circ} \mathrm{C}$ temperature ramp, which accuracy is shown in Fig. 6(b). The first prototype accuracy has been designed with a $150 \mathrm{MHz}$ tracking filter for a tradeoff between fast and efficient. This allows the carrier detection by looking for the maximum peak combined with the minimum energy in the near vicinity.

- 'Gt $\lambda$ ' (go to $\lambda$ ): This function provides wavelength allocation for the DFBs with an accuracy better of $\pm 1.5 \mathrm{GHz}$ to the target not to disturb adjacent channels. The LO wavelength will be tuned to catch the available OLT carrier, where the ONU receiver will be allocated; also is used for ONU TX tuning at the paired $\lambda$. Linear temperature jump requires about $5 \mathrm{~s}$. The accuracy for LO activation capturing the carrier is $\pm 400 \mathrm{MHz}$; and for the TX upstream at the paired $\lambda$ it is $\pm 1.5 \mathrm{GHz}$ (blind jump at $50 \mathrm{GHz}$ from the LO), which is below the push-in range of the LO AFC along the TW, and below the crosstalk guard band between adjacent channels. The function takes about $10 \mathrm{~s}$.

- 'DPC' (dynamic power control): increasing/decreasing $\pm 18 \mathrm{~dB}$ the DFB TX power, while keeping the same stable $\lambda$ without disturbing other transmitting channels. It manages the DFB temperature, tuning at $125 \mathrm{GHz} / \mathrm{V}$ slope with a conveniently linearized NTC, and simultaneously the bias current of the DFB at $2 \mathrm{GHz} / \mathrm{mA}$. This function lasts for about $50 \mathrm{~s}$ in this early prototype.

- 'AFC' (automatic frequency control): for LO tracking of the incoming wavelength, implementing an analogue proportionalintegral (PI) controller with accuracy better than $\pm 50 \mathrm{MHz}$ in a range of $3 \mathrm{GHz}( \pm 1.5 \mathrm{GHz})$. It performs fast tracking by bias current tuning, while the mean wavelength drift is corrected by thermal tuning. Moreover, it allows for low frequency FSK signaling up to hundreds of $\mathrm{kHz}$ by using the same AFC hardware (pilot tones) to assign the ONU-number and monitoring ONU TX center-channel [11].

With the available previous functions, the three required PON processes to perform the management and control for the flexible allocation are:

1) Initialization-activation process for the down/up channels: algorithm and sequence management to activate the first OLT, first
ONU and next demanding ONUs, establishing the communication channel, as illustrated in the basic flow diagram of Fig. 4:

The first step is to activate the OLT-carrier, fixing the downstream channel for the ONU-RX to detect the assigned wavelength and allocate the RX-LO. The OLT-carrier activation can be done at low power $(-18$ $\mathrm{dB}$ ) not disturbing neighbor channels by using the 'Gt $\lambda$ ' function and later the 'DPC' function to reach the operating optical power at the cost of an extra time, or can be done directly at high power with the ' $G \mathrm{t} \lambda$ ' function, which is faster (less than $5 \mu \mathrm{s}$ [33]), but at the cost of shorttime crosstalk (less than $700 \mathrm{~ns}$ [33]) with the neighbor channels. The OLT-RX is also active, ready to detect the ONU-TX when the ONU sends the upstream carrier. The OLT has always at least one down OLTcarrier available for a new ONU demanding connection.

When the ONU is being connected, it first detects the OLT-carrier with the 'BM' function and allocates the RX LO wavelength using the ' $\mathrm{G} \mathrm{t} \lambda$ ' function to go to the assigned channel, and finally uses the 'AFC' function to maintain less than $\pm 50 \mathrm{MHz}$ deviation error to the OLTcarrier for coherent demodulation of DPSK at $1.25 \mathrm{~Gb} / \mathrm{s}$. Once the downstream channel is fixed, the ONU-TX is allocated for the upstream at $50 \mathrm{GHz}$ up from the downstream channel. Due to the lack of integrated-paired DFBs, we use again the 'Gt $\lambda$ ' function at low power, and later the 'DPC' function to reach the bias power required for data transmission without changing the previously allocated wavelength.

Once the ONU is allocated, ONU-RX and TX are ready for activating the data communication: the ONU waiting for the OLT acknowledgment which consists in the downstream data activation. The OLT-RX has to detect the ONU-TX upstream with the 'BM' function for the OLT-LO to track the upstream carrier. Then, the OLT-LO, at operating optical power, is allocated to the ONU-TX wavelength with the 'Gt $\lambda$ ' function, and later the OLT-RX activates the 'AFC' function with the $\pm 50 \mathrm{MHz}$ maximum required tracking drift for coherent demodulation.

At first attempt the allocation is with $6.25 \mathrm{GHz}$ channel BW. The AFC function allows signaling with pilot tones, where the ONU informs the OLT about the desired bit rate and BW (e.g. 6.25, 12.5, $25 \mathrm{GHz}$ ) and also about the OLT-carrier position in its TW, and then the DWA algorithms at OLT can decide to keep or move the assigned OLT-carrier; and next, the OLT notifies the ONU at which final $\lambda$ it has to be allocated. Once the OLT-RX is ready, the downstream data and the upstream data transmissions are activated sequentially and the bidirectional link is established. If the ONU does not receives the acknowledgment from the OLT for a fixed period for any reason, e. g. a collision from another ONU trying to connect at the same time and same OLT-carrier, the ONU restarts this wavelength setting after a random delay period, which is a random procedure to overcome collisions that was proposed in [9].

During operation, the ONU LO is always tracking the downstream incoming channel with the 'AFC' function. Because of the paired integrated lasers, the ONU-TX with the upstream signal will maintain the relative wavelength at $50 \mathrm{GHz}$ from the LO. Meanwhile, the OLT is always monitoring the spectrum and can send FSK pilot tones, signaling with the ONU to guarantee long-term stabilization.

The whole link activation process takes less than 2 minutes: it is about $50 \mathrm{~s}$ for the downstream activation (LO allocation and AFC activation), less than $10 \mathrm{~s}$ for ONU-TX allocation at low power, and adding other $50 \mathrm{~s}$ for ONU-TX bias power and data activation.

2) ONU disconnection process: when one ONU wants to disconnect the communication channel it uses the 'DPC' function, dropping the power of the ONU-TX more than $18 \mathrm{~dB}$ from the operation transmission, in order to not disturb other neighbor channels. Disconnection takes about $200 \mu$ s and can sweep up to $120 \mathrm{GHz}$ into the band; then the OLT detects the upstream power drop and 
disconnects its TX, also with the 'DPC' function. The down and up channels are liberated and free for future ONUs connections.

3) ONU channel reallocation process: because of a fall-down channel, interference between channels or of flexible BW reassignment needs, the OLT can manage a channel to be moved to another $\lambda$. The MAC indicates the ONU to change the channel; then, the ONU and the OLT stop the 'AFC' function and disconnect their TXs using the 'DPC' function. The ONU-TX DFB reduces its power in $18 \mathrm{~dB}$; afterwards, the ONU LO sweeps the band searching for a new available OLT-carrier with the 'BM' function and finally the ONU-LO is tuned to the OLTcarrier with the ' $\mathrm{G} t \lambda$ ' function; then, the 'AFC' function is activated with the ONU-LO tracking the new down carrier. Once the down channel is established, the ONU-TX in low power transmission jumps to the 50 $\mathrm{GHz}$ paired channel using the 'Gt $\lambda$ ' function; afterwards, the ONU-TX increases its power to the operation transmission conditions using the 'DPC' function; lastly, the OLT-LO detects the ONU-TX with the 'BM' function and activates the 'AFC' tracking the upstream carrier. Finally, both sides data transmissions are activated.

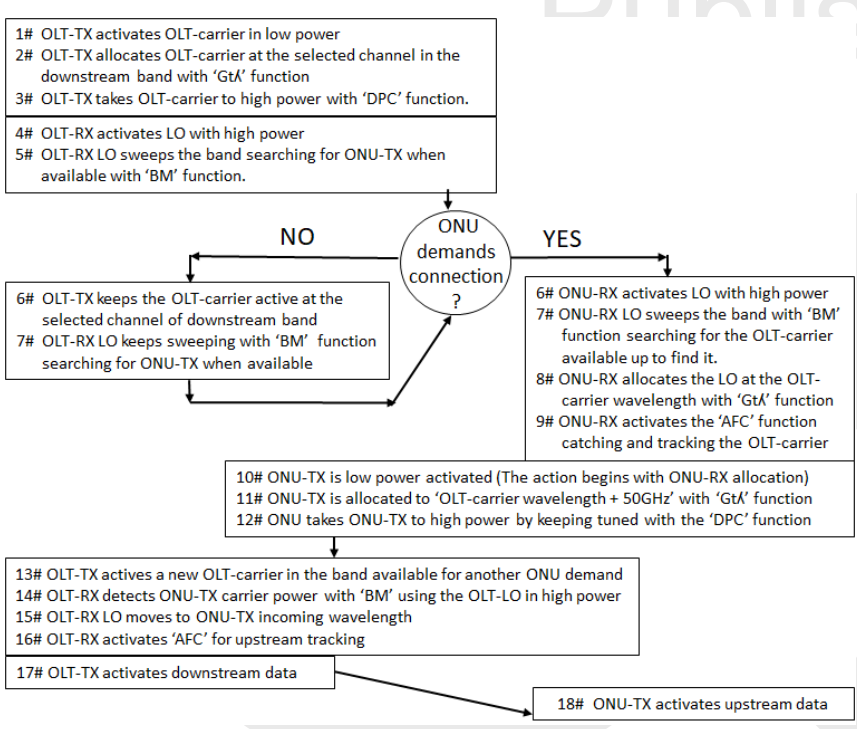

Fig. 4. ONU channel initialization-activation basic flow-diagram.

\section{EXPERIMENTAL RESULTS}

The activation process has been tested with 3 active channels at 1.25 $\mathrm{Gb} / \mathrm{s}$ which are neighbors and each one is centered in the middle of the $6.25 \mathrm{GHz}$ channel slot with a frequency drift better than $\pm 50 \mathrm{MHz}$ because of the AFC control. Fig. 5 presents the spectrum at the ONU side obtained from the 'BM' functionality (with $1.25 \mathrm{GHz}$ resolution OSA). The OLT-carrier provided by the OLT for the new incoming ONU to allocate the RX-LO is the highest level channel on the left. Three consecutive operating down channels are also present into the $50 \mathrm{GHz}$ sub-band.

Once the RX-LO is allocated to the OLT-carrier, the ONU activates the ONU-TX at the $50 \mathrm{GHz}$ paired channel; after, the OLT-RX detects the ONU-TX carrier and allocates the OLT-LO; then, the channels are ready for data activation, first for downstream and later for upstream.

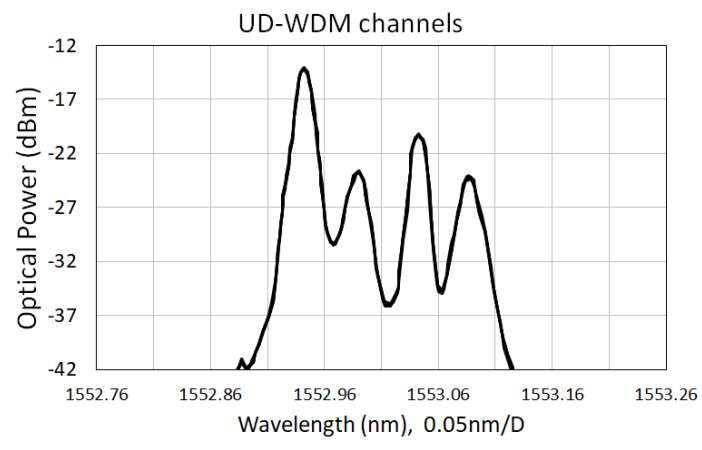

Fig. 5. Optical spectrum at ONU side with the down OLT-carrier (first on the left), and 3 active channels with downstream data (right).

Time measurements of the channel activation focused on frequency accuracy and time stabilization of the frequency allocation have been done with a preliminary prototype as proof of concept. The whole LO activation process always takes less than $50 \mathrm{~s}$ as reported. The activation process has been divided in several steps which are: 1) first of all, the "software initialization", which takes about $4 \mathrm{~s}$; 2) next, the "LO calibration", taking about $5.2 \mathrm{~s}$, where the LO laser is turned on at $30^{\circ} \mathrm{C}$; 3) then, follows "the initializing wavelength", which allocates the $\mathrm{LO}$ at the center of the $50 \mathrm{GHz}$ grid and takes $5.6 \mathrm{~s}$; 4) after, "scanning optical band", which takes about $12.2 \mathrm{~s}$, searching for the OLT-carrier; 5) then, comes the "allocation user wavelength", which fixes the LO over the incoming OLT-carrier, which takes an average of $15 s ; 6$ ) and finally, the "automatic frequency control" 'AFC' catches the OLT-carrier in about $600 \mathrm{~ms}$ and the LO keeps tracking the downstream signal. The LO activation average time has been tested to be about $42 \mathrm{~s}$, while the maximum is $46 \mathrm{~s}$. The activation process has been verified by temperature tuning with the 'Gt $\lambda$ ' function and depends on the wavelength stabilization time to the frequency target into the $50 \mathrm{GHz}$ grid. Some of the steps can be improved by stressing the hardware with the techniques described in reference [33] about DFB laser reallocation.

Once the LO is allocated, the upstream TX will be activated. Considering that both DFB lasers are paired, the ONU-TX activation follows next steps: 1) first of all, during the LO activation, the TX is tuned at $\mathrm{LO}+50 \mathrm{GHz}$ with low power not interfering other upstream channels, so this time is yet considered into the LO activation; 2) with the dynamic power control 'DPC' function, the TX laser increases the current typically $60 \mathrm{~mA}$ to reach $0 \mathrm{dBm}$ output power, while keeping the same wavelength. The 'DPC' function works increasing the bias current of the DFB while simultaneously cooling the DFB through the Peltier cell in several steps with a final frequency drift less than 1.5 GHz. 'DPC' average time is of $40 \mathrm{~s}$ and the maximum is $48 \mathrm{~s}$ for the channels in the shorter wavelength range; 3 ) finally, the 'AFC' control at the OLT side takes another $600 \mathrm{~ms}$ to catch and tracking the upstream channel. Hence, the total time for activation is under $100 \mathrm{~s}$ for this first prototype, and this times are in study for future improvement.

Once 'AFC' functions are running at both sides, the LOs are tracking the incoming signals with accuracy better than $50 \mathrm{MHz}$ and data demodulation is done with the performance presented in Fig. 7.

Fig. 6 illustrates the spectrum, at a photodiode output, of one OLTcarrier allocated between two downstream operating channels separated $12.5 \mathrm{GHz}$. Spectrum in Fig. 6 (a) is measured at a photodiode output with an ESA instrument, while the same spectrum measured with our 'BM' function, which works as a HR-OSA, is shown in Fig. 6 (b). 
(a)
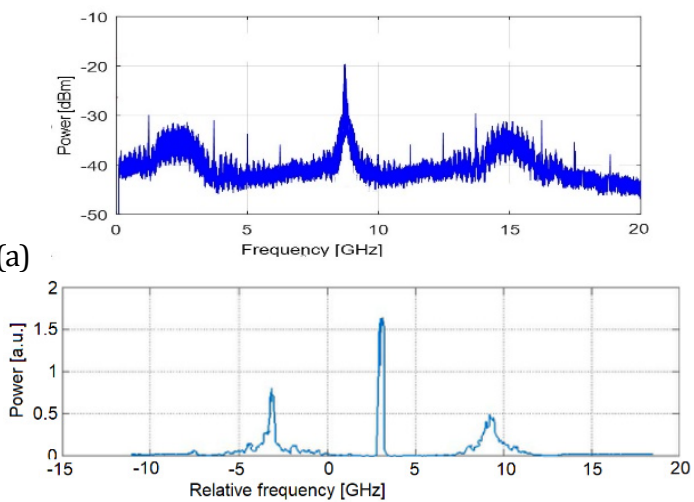

(b)

Fig. 6. Comparative of the heterodyned electrical spectra at ONU side: a) with external instrument and $\mathrm{LO}$ at $2.5 \mathrm{GHz}$ left from the first channel, b) with the implemented HR-OSA in the 'BM' function.

Sensitivity in terms of bit error rate (BER) vs. received power for 3photodiodes heterodyne receiver, 4-photodiodes heterodyne receiver, and 6-photodiodes homodyne polarization-independent receiver at $1.25 \mathrm{~Gb} / \mathrm{s}$, with $25 \mathrm{~km}$ single mode fiber spool and an optical attenuator emulating the split losses was evaluated, as depicted in Fig. 7. With a FEC threshold of BER $=10^{-3}$, the achieved sensitivities were 49.7 and $-49 \mathrm{dBm}$ for the heterodyne receivers with off-line postprocessing [27], and $-46 \mathrm{dBm}$ for the homodyne receiver realized with real-time DSP implemented in a commercial FPGA [32].

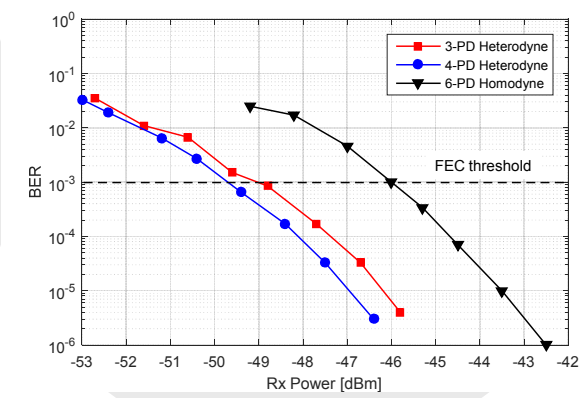

Fig. 7. Receiver sensitivity for polarization-independent coherent heterodyne and homodyne detection with $1.25 \mathrm{~Gb} / \mathrm{s}$ data rate.

The differences between homodyne and heterodyne receiver sensitivities mainly obey the different insertion losses of the optical front-end architectures, as well as the impairments like clock jitter and also synchronization mismatch, that become more apparent in a realtime DSP operation.

When crossing the neighbor channel by using the 'DPC' function, with the switching channel power attenuated $18 \mathrm{~dB}$, the SNR guarantees a BER penalty lower than $1 \mathrm{~dB}$ [34]. In fast activation process without attenuating the power of the switching channel, the effective crossing time takes less than $700 \mathrm{~ns}$, producing a burst error of 875 bits at $1.25 \mathrm{~Gb} / \mathrm{s}$ transmission, which can be assumed by buffering [33].

The obtained electrical spectra of two adjacent users at $\mathrm{Rb}=1.25$ $\mathrm{Gb} / \mathrm{s}$ for homodyne and heterodyne detection is shown in Fig 8. The channel separation for homodyne detection is of $6.25 \mathrm{GHz}$, as illustrated in Fig. 8 (a). When using heterodyne reception, the optical carriers between two users are separated $12.5 \mathrm{GHz}$, and the obtained electrical spectrum after heterodyning with an intermediate frequency (IF) of $2.5 \mathrm{GHz}$, is shown in Fig 8 (b). As the LO at $0 \mathrm{GHz}$ frequency reference has been allocated for heterodyne demodulation, it is at 2.5 $\mathrm{GHz}$ from the first user to be demodulated and at $10 \mathrm{GHz}$ from the closer neighbor and will be at $15 \mathrm{GHz}$ from the neighbor in the other side.

The homodyne receiver is more spectrally efficient than the low cost and off-the-self heterodyne one, but at the price of using ADCs.

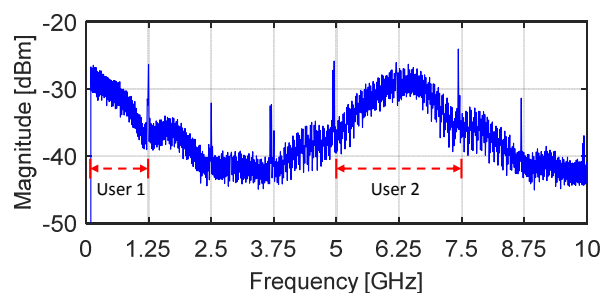

(a)

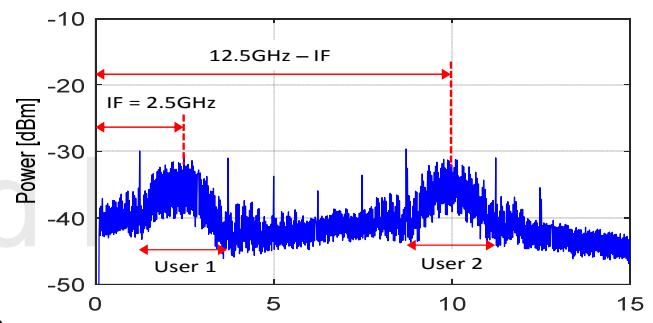

(b)

Frequency $[\mathrm{GHz}]$

Fig. 8. ONU electrical spectra after photo-detection of two users at 1.25 $\mathrm{Gb} / \mathrm{s}$ : (a) separated by $6.25 \mathrm{GHz}$ for homodyne detection; (b) separated by $12.5 \mathrm{GHz}$ for heterodyne detection, where the intermediate frequency (IF) is $2 \mathrm{x} R_{b}(2.5 \mathrm{GHz})$.

\section{CONCLUSION}

A wavelength-to-the-user UDWDM-PON with flexible efficient channel management has been proposed and tested for practical deployment. The system is based on low-cost coherent DFB transceivers with limited tunability, avoiding optical filters and providing high sensitivity. The splitting factor in the ODN can go from 32 up to 256. ONU and OLT architectures have been introduced with random DFB laser wavelengths with a statistical approach and channel assignment by heuristic DWA algorithms.

An experimental setup has been realized for ONU activation and operation processes. The basic optical band, organized in 8 down/up sub-bands of $50 \mathrm{GHz}$ serving 32 users of $6.25 \mathrm{GHz}$, can be scaled up to 256 ONUs with non-preselected wavelengths. For activation, the OLT provides a wavelength carrier to user's DFB LO to get connection; and, next, a paired DFB TX is blindly assigned. Experimental results show that high sensitivity and dense spectral allocation can be achieved.

Funding Information. Spanish Ministry of Science and Innovation, VERSONET work project (CICYT grant RTI 2018-97051).

\section{References}

1. 10G EPON standard IEEE P802.3av, 2009.

2. ITU-T G.987, “10-Gigabit-capable passive optical network (XG-PON) systems", (2012).

3. E. Agrell et al., "Roadmap of optical communications," Journal of Optics, 18, 1-40 (2016).

4. ITU-T, Recommendation G.989.2, “40-Gigabit-capable passive optical networks 2 (NGPON2): Physical media dependent (PMD) layer specification," (2014).

5. J.-H. Moon et al., "An automatic wavelength control method of a tunable laser for a WDM-PON," Photonics Technology Letters, 21, 325327 (2009). 
6. S.-R. Moon et al., "Automatic wavelength allocation method using Rayleigh Backscattering for a WDM-PON with tunable lasers", Journal of Optical Communications and Networking, 5, 190-197 (2013).

7. S. Pachnicke et al., "Tunable WDM-PON system with centralized wavelength control," Journal of Lightwave Technologies, 34, 812-818 (2015).

8. J. Zhu et al., "First demonstration of a WDM-PON system using full Cband tunable SFP+ transceiver modules," Journal of Optical Communications and Networking, 7, A28-A36 (2015).

9. H. Suzuki, "Colorless and plug-and-play technologies for WDM access over existing power-splitter-based infrastructure," Journal of Optical Networking, 6, 830-839 (2007).

10. J. Prat, I. Cano, M. Presi, I Tomkos, D. Klonidis, G. Vall-llosera, R. Brenot, R. Pous, G. Papastergiou, A. Rafel, and E. Ciaramella, "Technologies for cost effective udWDM-PONs," IEEE Journal of Lightwave Technology, 34, 783-791 (2016).

11. M. Presi et al., "Field-trial of a high-budget, filterless, $\lambda$-to-the-user, UDWDM-PON enabled by an innovative class of low-cost coherent transceivers," IEEE Journal of Lightwave Technology, 53, 5250-5259 (2017).

12. J. Segarra, V. Sales, and J. Prat, "Flexible coherent UDWDM-PON adapting dynamically to different bandwidths," in Proc. ICTON (2017), paper Tu.B2.6.

13. J. Segarra, V. Sales, and J. Prat, "Versatile metro-access network integrating FTTH, enterprises, IoT and $5 \mathrm{G}$ services," in Proc. ICTON (2019), paper Th.B1.1.

14. ITU-T, Recommendation G.694.1, "Spectral grids for WDM applications: DWDM frequency grid," (2012).

15. J. Tabares, S. Ghasemi, J. V. Velázquez, and J. Prat, "Coherent UltraDense WDM-PON enabled by complexity-reduced digital transceivers," Journal of Lightwave Technology, 38, 1305-1313 (2020).

16. J. Tabares, J. C. Velásquez, A. Napoli, and J. Prat, "Direct amplitudephase modulated dual-EML $10 \mathrm{~Gb} / \mathrm{s}$ optical transmitter," in Proc. ECOC, (2019), P59.

17. N. Cheng, G. Wei, and F. Effenberger, "Dynamic spectrum managed passive optical networks," IEEE Communications Magazine, 49, 86-93 (2011).

18. T. Garrod, F. Brunet, C. Galstad, M. Klaus, D. Olson, C. Zenner, Y. Xiao, L. Mawst, and D. Botez, "High-power and high-efficiency distributed feedback (DFB) lasers operating in the 1.4-1.6 $\mu \mathrm{m}$ range for eye-safe applications," Proc. SPIE, 8605, (2013).

19. I. N. Cano, A. Lerín, V. Polo, and J. Prat, "Direct phase modulation DFBs for cost-effective ONU transmitter in udWDM PONs," IEEE Photonics Technology Letters, 26, 973-975 (2014).

20. V. Sales, J. Segarra, V. Polo, J. C. Velásquez, and J. Prat, "UDWDM-PON using low-cost coherent transceivers with limited tunability and heuristic DWA" IEEE Journal of Optical Communications and Networking, 8, 582-599 (2016).

21. V. Sales, J. Segarra, V. Polo, and J. Prat, "Statistical UDWDM-PONs operating with ONU lasers under limited tunability," IEEE Photonics Technology Letters, 27, 257-260 (2015).

22. Y.-H. Lo et al., "'Tunable microwave generation of a monolithic dualwavelength distributed feedback laser," Optics Express, 22, 1312513137 (2014).

23. F. Pozzi, R. M. De La Rue, and M. Sorel, "Dual wavelength InAlGaAs-InP laterally coupled distributed feedback laser," IEEE Photonics Technology Letters, 18, 2563-2565 (2006).

24. M. Scheller et al., "High power dual-wavelength VECSEL based on a multiple folded cavity," IEEE Photonics Technology Letters, 29, 790-793 (2017).

25. Y.-D. Yang et al., "Tunable dual-wavelength coupled-cavity semiconductor lasers," in Proc. ECOC (2019), paper WP25.

26. C.-T. Tsai et al., "60-GHz millimeter-wave over fiber with directly modulated dual-mode laser diode," Scientific Reports, 6 (2016).
27. J. Tabares, V. Polo, and J. Prat," Polarization-independent heterodyne DPSK receiver based on $3 \times 3$ coupler for cost-effective udWDM PON, in Proc. OFC (2019), paper Th1K3.

28. J. Prat, V. Polo, P. Zakynthinos, I. Cano, J. A. Tabares, J. M. Fàbrega, D. Klonidis, and I. Tomkos, "Simple intradyne PSK system for udWDMPON," in Proc. ECOC (2012), paper We.2.B.2.

29. I. N. Cano, A. Lerín, V. Polo, and J. Prat, "Flexible D(Q)PSK 1.25-5 Gb/s UDWDM-PON with directly modulated DFBs and centralized polarization scrambling," in Proc. ECOC (2015), paper Th.1.3.7.

30. G. Y. Chu, A. Lerín, I. N. Cano, V. Polo, J. A. Tabares, and J. Prat "Exploiting RSOA for uplink transmission with coherent detection for low cost UDWDM-PON," in Proc. ACP (2014), paper AF2B.1.

31. M. P. McGarry, M. Reisslein, M. Maier, and K. Keha, "Bandwidth management for WDM PONs," Journal of Optical Networking, 5, 637654 (2006).

32. S. Ghasemi, J. Tabares, and J. Prat, "Symbol-rate digital signal processing for low-complexity polarization diversity intradyne optical PSK receivers," in Proc. ECOC (2019), P67.

33. V. Polo, P. Borotau, A. Lerin, and J. Prat, "DFB laser reallocation by thermal wavelength control for statistical udWDM in PONs," in Proc. ECOC (2014), paper P.4.13.

34. Ghasemi, S., Tabares, J., Polo, V., \& Prat, J. (2019). LUT-Free Carrier Recovery for Intradyne Optical DPSK Receivers in udWDM-PON. Journal of Lightwave Technology, 37(6), 1608-1613 